Pelin Batur, MD

Subspecialty Care for Women's Health, Ob/Gyn \& Women's Health Institute,

Cleveland Clinic; Associate Professor of Medicine, Cleveland Clinic Lerner

College of Medicine of Case Western Reserve University, Cleveland, $\mathrm{OH}_{\text {; }}$

Steering Committee, Women's Preventive Services Initiative, American

College of Obstetricians and Gynecologists and US Department of Health

and Human Services, Health Resources \& Services Administration; Working

Group Member, US Cervical Cancer Screening Risk Based Management

Guidelines Committee; Deputy Editor, Cleveland Clinic Journal of Medicine

\title{
The HPV vaccine: Understanding and addressing barriers to vaccination
}

I $\mathrm{N}$ THIS Issue, Drs. Vegunta and Long present an excellent review of conditions related to human papilloma virus (HPV), including cervical, vaginal, anal, oropharyngeal, and penile cancers. ${ }^{1}$

See related article, page 541

Cancers related to HPV have a major impact on a patient's life; even for those lucky enough to be cured, the morbidity associated with treatment can be considerable. Anatomic changes due to surgery may cause chronic pain and body image concerns, chemotherapy often leads to symptomatic premature menopause, and radiation treatment can cause anatomic changes to the genital tract, contributing to painful intercourse and sexual dysfunction. Treatment not only affects the patient physically, but impacts both the patient and partner psychologically, including plans for future childbearing.

It is not surprising that a clinician who cares for patients affected by HPV-related disease will be passionate about preventive efforts, especially encouraging HPV vaccination to healthy individuals during routine visits. We know that the HPV vaccine works. In 2018, a meta-analysis of 26 randomized controlled trials (including more than 70,000 women and girls) showed that the vaccine not only is well tolerated but significantly decreases the risk of preinvasive cervical disease in young women. 2,3 Additionally, there has been an $86 \%$ decrease in infection with the HPV subtypes causing cancers and genital warts doi:10.3949/ccjm.87a.20082 among teenage girls and a 40\% decrease in cervical precancers among vaccinated women. ${ }^{4}$

By decreasing infection with carcinogenic HPV strains, the hope is that the HPV vaccine may decrease the incidence of other HPV-related cancers, although there is not yet sufficient evidence to show this possible benefit.

We have a safe and effective cancer-prevention vaccine, but widespread vaccination remains a challenge in the United States. Patients may have concerns about vaccine safety and efficacy due to mixed messages from the media and other sources, but other common reasons for not vaccinating are provider discomfort in discussing sexuality, not receiving a strong recommendation from the clinician, and the belief by both the patient and provider that the patient is not at high risk. ${ }^{5}$

Similarly, a survey of guardians in a Texas school district (a state where the vaccination rate is $<50 \%$ ) identified scheduling conflicts and the lack of vaccine recommendation from a healthcare provider as significant hurdles to vaccination. ${ }^{6}$ Even in a New York school district that permitted adolescent vaccine selfconsent, scheduling and returning for the appointment were significant challenges affecting the vaccination rate. ${ }^{7}$

Key steps to implementing successful HPV vaccination in practice include understanding the individual patient's risk, following recommended vaccination timelines (which allow some flexibility with scheduling), and optimizing clinician-patient communication.

\section{We have a safe and effective cancer- prevention vaccine, but widespread vaccination remains a challenge}




\section{UNDERSTANDING HPV RISKS}

Any patient who is sexually active is at risk for HPV-related cancer. Approximately $80 \%$ of individuals will become infected with HPV at some point in their life, ${ }^{4}$ and HPV is the most common sexually transmitted disease in the United States. ${ }^{8}$

Yet despite how common HPV infection is, many women do not think that they are at risk. According to a survey of more than 900 unvaccinated females age 15 to 24, this belief was a main reason for forgoing vaccination (both in women who were and were not sexually active). ${ }^{9}$

For women who have sex with women, HPV can live on sexual devices for more than 24 hours. In addition to discussing the importance of vaccination, they should be counseled to use a barrier method over any shared sexual devices and clean the devices appropriately after each use. ${ }^{10}$

\section{WHO SHOULD RECEIVE THE VACCINE?}

In the United States, HPV vaccination is routinely recommended to all adults until age 26 , although the vaccine can be offered until
Any patient who is sexually active is at risk for HPV-related cancer age 45 in select patients who are not immunized. However, the vaccine is most likely to be beneficial when the series is completed before sexual debut; thus, guidelines recommend starting vaccination at age 11 to 12 , with the option to start as early as age $9 .{ }^{11}$

\section{COMMUNICATING EFFECTIVELY}

Clinicians may not feel comfortable discussing topics related to sexuality, especially in the field of pediatrics. A survey of members within 4 California chapters of the American Academy of Pediatrics showed that $71 \%$ of pediatricians would feel more comfortable discussing vaccination if the conversation also included education about HPV-related head and neck cancers. ${ }^{12}$

California Chapter 3 of the American Academy of Pediatrics created a 22-minute clinician training video that includes clinical vignettes of pediatricians counseling families who had concerns about vaccination (available at: https://aapca3.org/hpv-videos-education-promotion-project/). After watching this video, surveyed clinicians were shown to have improved their knowledge of vaccine safety, HPV disease burden (especially for males), and the importance of not delaying vaccination beyond preadolescence. ${ }^{13}$ This brief video intervention also led to more providers feeling "very comfortable" advising families. ${ }^{13}$

Another study looked at the most effective way to educate patients. A randomized controlled trial of 3 patient counseling strategies - an 8-minute educational video $(\mathrm{n}=$ 87), an educational handout with the same information written at an eighth-grade reading level $(n=84)$, and usual care $(n=85)$ was performed to assess vaccine acceptance. More patients in the educational video arm agreed to have the HPV vaccine $(51.7 \%)$ than in the handout or control arms $(33.3 \%$ and $28.2 \%$, respectively, $P<.01$ ). Interestingly, both the video and handout helped increase knowledge similarly, although the video helped most for the patient's decision to be vaccinated. ${ }^{14}$

In my practice, I start by notifying the patient that they are due for their HPV vaccine, just as I would do with any other preventive recommendation (eg, need for blood testing, cancer screening). Many patients will agree to this simple approach without a need for a long discussion about risks vs benefits, which may, paradoxically, lead to a greater hesitation to be vaccinated. ${ }^{15,16}$

Next, I clarify that the goal of HPV vaccination is to prevent cancer, and I remind the patient that everyone who engages in sexual activity is at risk. For those who have more concerns, it is important to first understand what the specific barriers are before trying to address them.

Despite our best efforts to educate, some patients may decline vaccination. It is important to avoid thinking of this as "losing a battle," as respecting patient autonomy ensures not only a trusted partnership in the patient's future healthcare, but also helps to minimize clinician frustration. Patients may opt to go against our recommendations in multiple situations, and they will make their own life decisions no matter how hard we try to provide optimal care. 


\section{COMPLETING THE VACCINATION SERIES}

Given that scheduling conflicts seem to play a prominent role in nonadherence to vaccination schedules, some flexibility with appointments is key. Anyone age 15 or older will need 3 doses of the HPV vaccine, typically with the second dose given 1 to 2 months after the first, and the third within 6 months of the first (at $0,1-2$, and 6 months). To accommodate a patient's schedule, the second HPV vaccination can be scheduled at 4 weeks after the first dose, and the third dose 12 weeks after the second. ${ }^{17}$ There should be at least 5 months between the first and third dose (referred to as the "minimal interval").

If repeat vaccination has occurred any earlier than these minimal intervals, then the patient will need another dose after the appropriate minimal interval has passed. Caution with interval dates is needed only when patients wish to come in earlier than recommended; if the vaccine schedule is interrupted or delayed, then a patient can continue with the remainder of the routine recommended schedule; no additional boosters or schedule adjustments are required (no maximum interval).

\section{SHOULD VACCINATION BE MANDATED?}

In Australia, where the HPV vaccine was made available for free in a national school program, vaccination rates of more than $70 \%$ have been achieved nationally in girls ages 12 to 13 , with resultant clinical benefits in preventing both warts and precancerous lesions. ${ }^{18} \mathrm{~A}$ meta-analysis of 9 high-income countries suggested that HPV infections decreased most when there is at least $50 \%$ coverage of the female population. ${ }^{19}$ In addition to Australia, Denmark, Canada (Quebec province), and New Zealand have offered widespread vaccination to multiple age cohorts, leading to optimized population immunity and the maximum impact on clinical outcomes. ${ }^{19,20}$

\section{REFERENCES}

1. Vegunta S, Long ME. Should women with human papillomavirus-related cancers be considered at high risk for cervical cancer?. Cleve Clin J Med 2020; 87(9)541-544. doi:10.3949/ccjm.87a.19145

2. Arbyn M, Xu L. Efficacy and safety of prophylactic HPV vaccines. A Cochrane review of randomized trials. Expert Rev Vaccines 2018; 17(12):1085-1091. doi:10.1080/14760584.2018.1548282
Vaccination rates vary greatly among US states. In contrast to the $78 \%$ vaccination rate in Washington, DC, which has a mandate for school entry, the Mississippi rate is only $29 \% .{ }^{20}$ As of 2019 , only 3 states provide free HPV vaccination through their health departments, although 25 states have laws requiring some funding for HPV education and vaccination. ${ }^{20}$

Of note, in the United States, there has been a higher uptake of the HPV vaccine among minority patients (higher in Hispanic and Black vs White populations) and also in those within a lower socioeconomic status group (income below federal poverty level, and having Medicaid coverage as opposed to private insurance). Because these populations of women historically have been at higher risk for HPV-related disease and cancer, it is hoped that this may lead to reversing some of these healthcare disparities. ${ }^{20}$

In the United States, significant controversy surrounds the idea of mandating vaccination prior to starting school, limiting vaccine exemptions, and the perceived loss of an individual's autonomy. Although a rise in vaccine-preventable illnesses has been seen primarily in communities with lower rates of vaccination, asking school administrators and nurses to "police" who is allowed to return to school adds an extra layer of complexity to this heated debate.

Although many may disagree with me, I suspect the best path to improving vaccination rates will not be achieved by adding more laws and rules, but by improving the education of both patients and caregivers, establishing a trusting patient-doctor relationship, simplifying office workflows (empowering nursing teams to educate patients, and then prompting clinicians that the vaccine order is needed), and by lifting financial barriers to vaccination, including copays, prior authorization, and coverage ambiguity.

\section{Clinicians may not feel comfortable discussing topics related to sexuality}

3. Arbyn M, Xu L, Simoens C, Martin-Hirsch PP. Prophylactic vaccination against human papillomaviruses to prevent cervical cancer and its precursors. Cochrane Database Syst Rev. 2018; 5(5):CD009069. doi:10.1002/14651858.CD009069.pub3

4. Centers for Disease Control and Prevention. Reasons to get vaccinated. https://www.cdc.gov/hpv/parents/vaccine/six-reasons.html. Accessed July 31, 2020.

5. Thompson EL, Rosen BL, Vamos CA, Kadono M, Daley EM. Human 
papillomavirus vaccination: what are the reasons for nonvaccination among US adolescents?. J Adolesc Health 2017; 61(3):288-293. doi:10.1016/j.jadohealth.2017.05.015

6. Victory M, Do TQN, Kuo YF, Rodriguez AM. Parental knowledge gaps and barriers for children receiving human papillomavirus vaccine in the Rio Grande Valley of Texas. Hum Vaccin Immunother 2019; 15(7-8):1678-1687. doi:10.1080/21645515.2019.1628551

7. Oliver K, McCorkell C, Pister I, Majid N, Benkel DH, Zucker JR. Improving HPV vaccine delivery at school-based health centers. Hum Vaccin Immunother 2019; 15(7-8):1870-1877. doi:10.1080/21645515.2019.1578596

8. Centers for Disease Control and Prevention. Genital HPV infectionfact sheet. Accessed July 31, 2020. https://www.cdc.gov/std/hpv/ stdfact-hpv.htm.

9. Liddon NC, Hood JE, Leichliter JS. Intent to receive HPV vaccine and reasons for not vaccinating among unvaccinated adolescent and young women: findings from the 2006-2008 National Survey of Family Growth. Vaccine 2012; 30(16):2676-2682. doi:10.1016/j.vaccine.2012.02.007

10. Rubin ES, Deshpande NA, Vasquez PJ, Kellogg Spadt S. A clinical reference guide on sexual devices for obstetrician-gynecologists. Obstet Gynecol 2019; 133(6):1259-1268. doi:10.1097/AOG.0000000000003262

11. Meites E, Szilagyi PG, Chesson HW, Unger ER, Romero JR, Markowitz LE. Human papillomavirus vaccination for adults: updated recommendations of the Advisory Committee on Immunization Practices. MMWR Morb Mortal Wkly Rep 2019; 68(32):698-702. doi:10.15585/mmwr.mm6832a3

12. Berger MH, Haidar YM, Bitner B, Trent M, Tjoa T. Practice patterns and knowledge among California pediatricians regarding human papillomavirus and its relation to head and neck cancer. Am J Oto- laryngol 2019; 40(4):525-529. doi:10.1016/j.amjoto.2019.04.011

13. Kumar MM, Boies EG, Sawyer MH, Kennedy M, Williams C, Rhee KE. A brief provider training video improves comfort with recommending the human papillomavirus vaccine. Clin Pediatr (Phila) 2019; 58(1):17-23. doi:10.1177/0009922818805217

14. Cory L, Cha B, Ellenberg S, et al. Effects of educational interventions on human papillomavirus vaccine acceptability: a randomized controlled trial. Obstet Gynecol 2019; 134(2):376-384. doi:10.1097/AOG.0000000000003379

15. Zhang S, Batur P. Human papillomavirus in 2019: an update on cervical cancer prevention and screening guidelines. Cleve Clin J Med 2019; 86(3):173-178. doi:10.3949/ccjm.86a.18018

16. Zhang S, Batur P. In reply: human papillomavirus. Cleve Clin J Med 2019; 86(5):300-301. doi:10.3949/ccjm.86c.05002

17. Centers for Disease Control and Prevention. Recommended child and adolescent immunization. Accessed August 17, 2020. https:// www.cdc.gov/vaccines/schedules/downloads/child/0-18yrs-combinedschedule-bw.pdf

18. Garland SM. The Australian experience with the human papillomavirus vaccine. Clin Ther 2014; 36(1):17-23. doi:10.1016/j.clinthera.2013.12.005

19. Drolet M, Bénard É, Boily MC, et al. Population-level impact and herd effects following human papillomavirus vaccination programmes: a systematic review and meta-analysis. Lancet Infect Dis 2015; 15(5):565-580. doi:10.1016/S1473-3099(14)71073-4

20. Dilley S, Miller KM, Huh WK. Human papillomavirus vaccination: ongoing challenges and future directions. Gynecol Oncol 2020; 156(2):498-502. doi:10.1016/j.ygyno.2019.10.018

Address: Pelin Batur, MD, Women's Health Institute, A8-406, Cleveland Clinic, 9500 Euclid Avenue, Cleveland, OH 44195; baturp@ccf.org 